\title{
Dynamical Systems with Multivalued Integrals on a Torus
}

\author{
V. V. Kozlov ${ }^{a}$ \\ Received August 2006
}

\begin{abstract}
Properties of the solutions to differential equations on the torus with a complete set of multivalued first integrals are considered, including the existence of an invariant measure, the averaging principle, and the infiniteness of the number of zeros for integrals of zero-mean functions along trajectories. The behavior of systems with closed trajectories of large period is studied. It is shown that a generic system acquires a limit mixing property as the periods tend to infinity.
\end{abstract}

DOI: $10.1134 /$ S0081543807010105

\section{INTRODUCTION. MULTIVALUED INTEGRALS}

Let $\mathbb{T}^{n}=\left\{x_{1}, \ldots, x_{n} \bmod 1\right\}$ be the $n$-torus with angular coordinates $\left(x_{1}, \ldots, x_{n}\right)=x$, and let

$$
\dot{x}_{1}=v_{1}\left(x_{1}, \ldots, x_{n}\right), \ldots, \dot{x}_{n}=v_{n}\left(x_{1}, \ldots, x_{n}\right)
$$

be differential equations on $\mathbb{T}^{n}$ (the functions $v_{1}, \ldots, v_{n}$ are 1 -periodic in each coordinate $x_{1}, \ldots, x_{n}$ ) with sufficiently smooth right-hand sides. The meaning of "sufficiently" is to be rendered more precise; at least, the results stated below are valid for infinitely differentiable functions $v_{1}, \ldots, v_{n}$.

By a multivalued integral of system (1.1) we understand a closed (but not exact) 1 -form $\varphi$ on $\mathbb{T}^{n}$ such that

$$
i_{v} \varphi=0
$$

where $v=\left(v_{1}, \ldots, v_{n}\right)$. Locally, $\varphi=d H$, and

$$
\dot{H}=\sum \frac{\partial H}{\partial x_{j}} v_{j}=i_{v} d H=0
$$

according to (1.2). Thus, locally, the function $H$ is an ordinary integral of system (1.1). On the $n$-space $\mathbb{R}^{n}=\left\{x_{1}, \ldots, x_{n}\right\}$ covering the torus $\mathbb{T}^{n}$, the closed 1 -form $\varphi$ is exact, i.e., $\varphi=d H$, where $H$ is a single-valued function on $\mathbb{R}^{n}$.

Example. Suppose that $n=2$ and system (1.1) has an integral invariant

$$
\iint \rho\left(x_{1}, x_{2}\right) d x_{1} d x_{2}
$$

where $\rho$ is a positive smooth function on $\mathbb{T}^{2}$. The invariance condition reduces to the Liouville equation

$$
\frac{\partial\left(\rho v_{1}\right)}{\partial x_{1}}+\frac{\partial\left(\rho v_{2}\right)}{\partial x_{2}}=0
$$

\footnotetext{
${ }^{a}$ Steklov Institute of Mathematics, Russian Academy of Sciences, ul. Gubkina 8, Moscow, 119991 Russia.

E-mail address: kozlov@pran.ru
} 
For this system, the differential 1-form

$$
\varphi=-\rho v_{2} d x_{1}+\rho v_{1} d x_{2}
$$

is a multivalued integral. As a rule, one considers the case when system (1.1) has no equilibrium states; then, the 1 -form (1.3) is never exact on $\mathbb{T}^{2}$.

Differential equations on the two-dimensional torus with an integral invariant and without singular points were studied by Poincaré [1] and Kolmogorov [2, 3]. The purpose of this paper is to extend their classical results to the multidimensional case. The first results in this direction were obtained by Arnold [4]. In [5, 6], Hamiltonian systems with complete sets of multivalued integrals were considered.

Let $\Gamma_{1}, \ldots, \Gamma_{n}$ be a "canonical" system of basic 1-cycles on $\mathbb{T}^{n}$, each of which once encircles the torus:

$$
\int_{\Gamma_{j}} d x_{i}=\delta_{i j}
$$

where $\delta_{i j}$ is the Kronecker delta. It is natural to call the numbers

$$
a_{j}=\int_{\Gamma_{j}} \varphi, \quad 1 \leq j \leq n,
$$

the periods of the multivalued "function" $\varphi$. If $\varphi=d H$, then

$$
H=a_{1} x_{1}+\ldots+a_{n} x_{n}+h\left(x_{1}, \ldots, x_{n}\right),
$$

where $h$ is a function on $\mathbb{T}^{n}$ (which is 1 -periodic in $x_{1}, \ldots, x_{n}$ ). We refer to the function $H$ as a multivalued integral of system (1.1) on $\mathbb{T}^{n}$ as well.

We study systems (1.1) that have $n-1$ independent multivalued integrals $H_{2}, \ldots, H_{n}$. Such systems are called polyintegrable in [4]; physicists call them Nambu systems [7]. As S.P. Novikov noticed, studying the so-called geometric limit of a strong magnetic field reduces to the analysis of some special Hamiltonian systems with a multivalued Hamiltonian on Fermi surfaces (see surveys [8, 9]).

According to (1.4), we have

$$
H_{i}(x)=a_{i 1} x_{1}+\ldots+a_{i n} x_{n}+h_{i}\left(x_{1}, \ldots, x_{n}\right) ;
$$

here,

$$
a_{i j}=\int_{\Gamma_{j}} d H_{i} .
$$

Let $H_{1}$ be another multivalued function on $\mathbb{T}^{n}$, and let $A=\left\|a_{i j}\right\|$ be the period matrix of the 1 -forms $d H_{1}, d H_{2}, \ldots, d H_{n}$. The matrix $A$ is obtained from the Jacobi matrix of the functions $H_{1}, H_{2}, \ldots, H_{n}$ by averaging over $\mathbb{T}^{n}$. The further considerations are based on the following theorem.

Theorem 1. Suppose that $\operatorname{det} A \neq 0$ and

$$
\frac{\partial\left(H_{1}, H_{2}, \ldots, H_{n}\right)}{\partial\left(x_{1}, x_{2}, \ldots, x_{n}\right)} \neq 0
$$

everywhere on $\mathbb{T}^{n}$. Then, an invertible change of variables $x \mapsto u \bmod 1$ transforms system (1.1) into the system

$$
\dot{u}_{j}=\alpha_{j} r\left(u_{1}, \ldots, u_{n}\right), \quad 1 \leq j \leq n,
$$

where $\alpha_{j}=$ const and $r: \mathbb{T}^{n} \rightarrow \mathbb{R}$. 
Proof. We set

$$
\begin{gathered}
u_{1}=\alpha_{1} H_{1}+\beta_{1} H_{2}+\ldots+\gamma_{1} H_{n}, \\
\ldots \ldots \ldots \ldots \ldots \ldots \ldots \ldots+\gamma_{n} \ldots \ldots+\gamma_{n} H_{n}, \\
u_{n}=\alpha_{n} H_{1}+\beta_{n} H_{2}+\ldots+
\end{gathered}
$$

where the vectors

$$
\varepsilon_{1}=\left(\alpha_{1}, \beta_{1}, \ldots, \gamma_{1}\right)^{T}, \ldots, \varepsilon_{n}=\left(\alpha_{n}, \beta_{n}, \ldots, \gamma_{n}\right)^{T}
$$

are defined by the relations

$$
A^{T} \varepsilon_{1}=\mathrm{e}_{1}, \ldots, A^{T} \varepsilon_{n}=\mathrm{e}_{n}
$$

with $\mathrm{e}_{1}=(1,0, \ldots, 0)^{T}, \ldots, \mathrm{e}_{n}=(0,0, \ldots, 1)^{T}$ being the basis vectors in $\mathbb{R}^{n}$. Since the period matrix $A$ is nondegenerate, the coefficients in (1.8) are determined uniquely.

Taking into account formulas (1.5) and (1.9), we can represent the transformation (1.8) in the form

$$
\begin{gathered}
u_{1}=x_{1}+\alpha_{1} h_{1}+\ldots+\gamma_{1} h_{n}, \\
\ldots \ldots \ldots \ldots \ldots \ldots+\ldots+\gamma_{n} h_{n} . \\
u_{n}=x_{n}+\alpha_{n} h_{1}+\ldots+\gamma .
\end{gathered}
$$

Using (1.5) and (1.9), it is easy to prove that the transformation (1.8) (or, equivalently, (1.10)) is nondegenerate:

$$
\frac{\partial\left(u_{1}, \ldots, u_{n}\right)}{\partial\left(x_{1}, \ldots, x_{n}\right)}=(\operatorname{det} A)^{-1} \frac{\partial\left(H_{1}, \ldots, H_{n}\right)}{\partial\left(x_{1}, \ldots, x_{n}\right)} \neq 0
$$

(see the assumption (1.6)). In particular, (1.10) defines a locally invertible mapping of $\mathbb{T}^{n}$ onto itself. Note that the transformation (1.10), when treated as a self-mapping of $\mathbb{R}^{n}$, takes the lattice $\mathbb{Z}^{n}$ to itself (up to a translation). Therefore, formula (1.10) specifies an automorphism of $\mathbb{T}^{n}$.

Since $H_{2}, \ldots, H_{n}$ are first integrals of (1.1), it follows that, in the new variables $u_{1}, \ldots, u_{n}$ mod 1, equations (1.1) take the form

$$
\dot{u}_{j}=\alpha_{j} \dot{H}_{1}=\alpha_{j} r(u),
$$

where

$$
r(u)=\left.i_{v} d H_{1}\right|_{u}
$$

as required.

The degree of smoothness of the transformation rectifying the trajectories of system (1.1) coincides with that of the periodic functions $h_{1}, \ldots, h_{n}$. The rectifiability of the trajectories of a polyintegrable vector field on the three-dimensional torus was proved by Arnold under the assumption that there are no singular points [4]. For $n=2$, the condition $v \neq 0$ implies that, in some angular coordinates on $\mathbb{T}^{2}$, we have

$$
v_{1}(x) \neq 0 .
$$

This fact is usually proved by applying the well-known Siegel's theorem about integral curves on the 2-torus [10] under the assumption that the system has an integral invariant. For $n=2$, the rectifiability of trajectories was proved in [2]. In the multidimensional case, Siegel's theorem is, of course, false. We emphasize that the absence of singular points is not assumed in Theorem 1. 
In what follows, we study system (1.1) under the assumption that $v(x) \neq 0$ everywhere on $\mathbb{T}^{n}$. If the conditions of Theorem 1 hold, then the vector field (1.7) has no singular points either. Therefore, $\sum \alpha_{j}^{2} \neq 0$, and the function $r=i_{v} d H_{1}$ vanishes nowhere. This implies, in particular, that (under the conditions of Theorem 1) in the absence of singular points, there exist angular coordinates on $\mathbb{T}^{n}$ in which (1.13) holds.

It turns out that system (1.1) has an integral invariant

$$
\int \rho(x) d^{n} x
$$

with density

$$
\rho=\frac{1}{i_{v} d H_{1}} \frac{\partial\left(H_{1}, \ldots, H_{n}\right)}{\partial\left(x_{1}, \ldots, x_{n}\right)} .
$$

Let us prove this simple fact. Consider the differential $n$-form

$$
\Omega=\frac{1}{i_{v} d H_{1}} d H_{1} \wedge d H_{2} \wedge \ldots \wedge d H_{n}=\rho d x_{1} \wedge \ldots \wedge d x_{n} .
$$

Since it is closed (as any $n$-form on $n$-space), its Lie derivative equals $d i_{v} \Omega$. On the other hand,

$$
i_{v} \Omega=d H_{2} \wedge \ldots \wedge d H_{n}-\frac{1}{i_{v} d H_{1}} d H_{1} \wedge\left(i_{v} d H_{2}\right) \wedge \ldots \wedge d H_{n}+\ldots=d H_{2} \wedge \ldots \wedge d H_{n} .
$$

It remains to note that this form is closed.

Having the invariant measure (1.14), we can average the right-hand sides of system (1.1). As a result, we obtain a simplified averaged system with constant vector field

$$
\dot{x}_{i}=\omega_{i}, \quad \omega_{i}=\int_{\mathbb{T}^{n}} v_{i} \rho d^{n} x / \int_{\mathbb{T}^{n}} \rho d^{n} x .
$$

Of course, the averaging principle, which consists in replacing (1.1) by (1.16), must be justified. Theorem 1 can be refined as follows.

Theorem 1'. Suppose that the conditions of Theorem 1 hold and $i_{v} d H \neq 0$. Then, $\alpha_{j}=c \omega_{j}$, where $c=$ const $\neq 0$.

Curiously, in the first work by Kolmogorov [2] (where the case $n=2$ was considered), nothing was said about this. However, in [3], Kolmogorov stated a theorem on the rectification of trajectories together with the equality $\alpha_{j}=c \omega_{j}$.

Proof of Theorem $\mathbf{1}^{\prime}$. We use the simple formula

$$
v_{j}=\sum \frac{\partial x_{j}}{\partial u_{k}} \dot{u}_{k}=\left(\sum \frac{\partial x_{j}}{\partial u_{k}} \alpha_{k}\right) r .
$$

On the other hand, relation (1.11) readily implies

$$
\rho(x) d^{n} x=\frac{|A|^{-1}}{r(u)} d^{n} u
$$

Therefore, substituting (1.17) and (1.18) into (1.16), we obtain

$$
\omega_{j}=\int_{\mathbb{T}^{n}}\left(\sum \frac{\partial x_{j}}{\partial u_{k}} \alpha_{k}\right) d^{n} u / \int_{\mathbb{T}^{n}} \frac{d^{n} u}{r(u)} .
$$


Inverting (1.10), we obtain simple formulas $x_{j}=u_{j}+X_{j}$, where $X_{j}$ are 1-periodic functions of $u_{1}, \ldots, u_{n}$. Since

$$
\int_{\mathbb{T}^{n}} \frac{\partial X_{j}}{\partial u_{k}} d^{n} u=0
$$

it follows that

$$
\int_{\mathbb{T}^{n}}\left(\sum \frac{\partial x_{j}}{\partial u_{k}} \alpha_{k}\right) d^{n} u=\sum \delta_{j k} \alpha_{k}=\alpha_{j} .
$$

Thus, (1.19) implies the required formula

$$
\omega_{j}=\alpha_{j} / \int_{\mathbb{T}^{n}} \frac{d^{n} u}{r},
$$

which proves the theorem.

Formula (1.20) allows us to reformulate Theorems 1 and $1^{\prime}$ as follows: System (1.1) reduces to the system

$$
\dot{u}_{j}=\omega_{j} \widetilde{r}\left(u_{1}, \ldots, u_{n}\right), \quad 1 \leq j \leq n,
$$

and the invariant measure $d^{n} u /|\widetilde{r}|$ is a probability measure:

$$
\int_{\mathbb{T}^{n}} \frac{d^{n} u}{|\widetilde{r}|}=1
$$

\section{GENERAL PROPERTIES OF SYSTEMS WITH MULTIVALUED INTEGRALS}

We will examine the system of differential equations (1.7) under the condition $r \neq 0$. For $n=2$, these equations have long been studied by many authors, including Poincaré [1]. System (1.7) has an integral invariant with density

$$
R(u)=\frac{1}{r(u)} .
$$

We say that "frequencies" $\omega_{1}, \ldots, \omega_{n}$ are incommensurable if the resonance relation

$$
\sum k_{j} \omega_{j}=0
$$

with integer $k_{j}$ holds only for $k_{1}=\ldots=k_{n}=0$. These frequencies are said to be strongly incommensurable if, for all integer vectors $k \neq 0$, we have

$$
|(\omega, k)| \geq \lambda|k|^{-\nu}
$$

where $\lambda$ and $\nu$ are positive constants. It is well known that the power estimate (2.1) is valid for almost all vectors $\omega \in \mathbb{R}^{n}$.

Theorem 2. Suppose that $R: \mathbb{T}^{n} \rightarrow \mathbb{R}$ is an infinitely differentiable (analytic) function and the frequencies $\omega_{j}\left(\right.$ or $\left.\alpha_{j}\right)$ are strongly incommensurable. Then, there exists an invertible infinitely differentiable (analytic) change of variables $u \mapsto w \bmod 1$ that reduces system (1.7) to the form

$$
\dot{w}_{j}=\omega_{j}, \quad 1 \leq j \leq n .
$$

This is an extension of the well-known Kolmogorov's theorem [2, 3] to the multidimensional case. Theorem 2 justifies the averaging principle from Section 1 in the case of strongly incommensurable frequencies. 
Theorem 2 was proved in [11] in a somewhat different form. A similar assertion for flows of finite and infinite smoothness was proved later by Herman in [12]. The proofs given in [11] and [12] are conceptually the same.

We say that system (1.7) reduces to the form (2.2) by an invertible continuous transformation if there exists a homeomorphism $\psi: \mathbb{T}^{n} \rightarrow \mathbb{T}^{n}$ such that the diagram

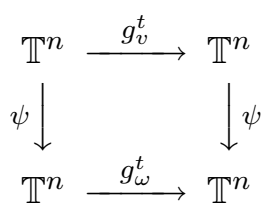

is commutative for all values of time $t$. Here, $g_{v}^{t}$ and $g_{\omega}^{t}$ are the phase flows of the differential equations (1.7) and (2.2), respectively.

If $\psi$ is a continuously differentiable transformation, then the commutativity of diagram (2.3) means that the substitution $w=\psi(u)$ transforms the system of equations (1.7) into system (2.2).

Theorem 3. Suppose that the frequencies $\omega_{1}, \ldots, \omega_{n}$ are incommensurable and system (1.7) reduces to the form (2.2) by an invertible continuous transformation. Then, the integral

$$
\int_{0}^{\tau}\left[R\left(\omega_{1} t, \ldots, \omega_{n} t\right)-\langle R\rangle\right] d t, \quad\langle R\rangle=\int_{\mathbb{T}^{n}} R(u) d^{n} u,
$$

is bounded as a function of the upper limit $\tau$.

It is well known that in this case (2.4) is a conditionally periodic function of $\tau$. Otherwise, the required reduction is impossible. It is this form in which Poincaré stated his conjecture on the irreducibility of system (1.7) [1].

The irreducibility condition can be formulated in a somewhat different form. Let

$$
\sum R_{m} e^{2 \pi i(m, u)}, \quad m \in \mathbb{Z}^{n}
$$

be the Fourier series of the invariant-measure density $R$.

Theorem 4. If $\omega_{1}, \ldots, \omega_{n}$ are incommensurable and

$$
\sum^{\prime}\left|\frac{R_{m}}{(m, \omega)}\right|^{2}=\infty
$$

then there exists no invertible continuous transformation that would reduce system (1.7) to the form (2.2).

Condition (2.5) is a continuous analog of the divergence condition for series (8) from [2]. Refinements and strengthenings of Kolmogorov's reducibility theorem (for $n=2$ ) are given in [13-15]. Conditions for the reducibility and irreducibility of system (1.7) to the form (2.2) (and their discrete analogs) in the case of strongly incommensurable frequencies (to be more precise, in the case when the vector with components $\left(\omega_{1} / \omega_{n}, \ldots, \omega_{n-1} / \omega_{n}\right)$ is Diophantine) can be found in $[16,17]$.

Let us return to the initial system (1.1) with multivalued integrals. Suppose that the conditions of Theorems 1 and $1^{\prime}$ hold. Let $g^{t}$ be the phase flow of system (1.1), and let $\rho$ again denote the density (1.15) of the integral invariant.

Theorem 5. If $\omega_{1}, \ldots, \omega_{n}$ are incommensurable and $f$ is a Riemann integrable function on $\mathbb{T}^{n}$, then

$$
\lim _{\tau \rightarrow \infty} \frac{1}{\tau} \int_{0}^{\tau} f\left(g^{t}(x)\right) d t=\int_{\mathbb{T}^{n}} f \rho d^{n} x / \int_{\mathbb{T}^{n}} \rho d^{n} x
$$

uniformly in $x$. 
This is a simple generalization of the classical Weyl's theorem on uniform distribution. Of course, it is assumed here that the function $t \mapsto f\left(g^{t}(x)\right)$ is Riemann integrable for $t \geq t_{0}$ and all $x \in \mathbb{T}^{n}$. Among other things, Theorem 5 implies that for incommensurable $\omega_{1}, \ldots, \omega_{n}$, the phase flow of system (1.1) is strictly ergodic. If the frequencies $\omega_{1}, \ldots, \omega_{n}$ are commensurable, then the limit on the left-hand side of (2.6) exists for all $x$; this limit is a Riemann integrable function $\bar{f}(x)$ invariant with respect to the phase flow $g^{t}$ and satisfying the relation

$$
\int_{\mathbb{T}^{n}} \bar{f} \rho d^{n} x=\int_{\mathbb{T}^{n}} f \rho d^{n} x .
$$

Theorem 6. Let $f$ be a continuous function on the torus and $\langle f\rangle$ be the mean on the right-hand side of (2.6). Then, there exists an $x \in \mathbb{T}^{n}$ such that

(1) $f(x)=\langle f\rangle$;

(2) $\int_{0}^{\tau} f\left(g^{t}(x)\right) d t-\langle f\rangle \tau \geq 0(\leq 0)$ for all $\tau \in \mathbb{R}$.

We emphasize that the frequencies $\omega_{1}, \ldots, \omega_{n}$ are not assumed to be rationally incommensurable. Theorem 6 generalizes and strengthens the well-known Bohl's theorem on the integrals of conditionally periodic functions [18].

Theorem 7. Suppose that $f, h_{1}, \ldots, h_{n}, i_{v} d H_{1}$ are infinitely differentiable functions on $\mathbb{T}^{n}$, the frequencies $\omega_{1}, \ldots, \omega_{n}$ are incommensurable, and $f(x) \neq\langle f\rangle$. Then, the function

$$
\tau \mapsto \int_{0}^{\tau} f\left(g^{t}(x)\right) d t-\langle f\rangle \tau
$$

has infinitely many zeros as $\tau \rightarrow \infty$.

Apparently, Theorem 7 is also valid for functions with a finite smoothness degree that depends on $n$. For example, in the case of $n=2, C^{1}$ smoothness is sufficient. However, for continuous $f$ Theorem 7 is false. In [19], a counterexample for $n=2$ was constructed, in which system (1.1) has a very simple form: $\dot{x}_{1}=1, \dot{x}_{2}=\sqrt{2}$.

For completeness, we state another theorem on the oscillations of the function (2.7).

Theorem 8. Suppose that $f$ is a Lebesgue integrable function and the frequencies $\omega_{1}, \ldots, \omega_{n}$ are incommensurable. Then, for almost all (with respect to the Lebesgue measure) $x \in \mathbb{T}^{n}$, the function (2.7) changes sign infinitely many times as $\tau \rightarrow \infty$ in the weak sense, i.e., this function cannot be positive or negative starting from some $\tau$.

An example constructed in [19] shows that even for a continuous function $f$, the exceptional set of points in $\mathbb{T}^{n}$ may be dense in the torus.

\section{DIFFUSION AND LIMIT MIXING}

In this section, we continue the study of system (1.7) to which the original system (1.1) reduces under the assumptions of Theorem 1 . Let $F$ and $G$ be arbitrary square integrable (with respect to the invariant measure $d \mu=R(u) d^{n} u$ ) functions on $\mathbb{T}^{n}$, and let $g^{t}$ be the phase flow of system (1.7). We set

$$
K(t)=\int_{\mathbb{T}^{n}} F\left(g^{t}(u)\right) G(u) R(u) d^{n} u .
$$

If this function tends to

$$
\int_{\mathbb{T}^{n}} F R d^{n} u \int_{\mathbb{T}^{n}} G R d^{n} u / \int_{\mathbb{T}^{n}} R d^{n} u
$$

as $t \rightarrow \infty$, then system (1.7) has the mixing property. 
The mixing properties of system (1.7) for $n=2$ were studied already by Poincaré [1], who gave, in particular, a precise definition of systems with mixing. He also conjectured that the system has the mixing property if the integral (2.4) is unbounded. In this case (by Theorem 3), system (1.7) cannot be reduced to a system of the form (2.2), and the latter is obviously not mixing.

Poincaré's conjecture was disproved for $n=2$ in [20,21] by using cyclic approximations. The stronger assertion that the system has the uniform recurrence property in the two-dimensional case was proved in [19]: If $R \in C^{1}$ and $\alpha_{2} / \alpha_{1}$ is irrational, then there exists an unbounded sequence of time points $t_{n}$ such that

$$
\left\|g^{t_{n}}(x)-x\right\| \rightarrow 0
$$

as $n \rightarrow \infty$ uniformly in $x$. On the other hand, if the function $r$ in (1.7) has a singularity, then mixing may occur even for $n=2[22,23]$.

We say that system (1.7) has the diffusion property if, for all $F, G \in L_{2}\left(\mathbb{T}^{n}\right)$, the function (3.1) has a limit as $t \rightarrow \infty$ (which does not necessarily coincide with (3.2)). To better understand this definition, consider the case when $F$ is the density of a probability distribution on the torus, i.e., $F \geq 0$ and

$$
\int_{\mathbb{T}^{n}} F R d^{n} u=1 .
$$

Let $G$ be the characteristic function of a measurable domain $\mathcal{D} \subset \mathbb{T}^{n}$. Then, $K(t)$ is the probability that the system is in the domain $\mathcal{D}$ at time $-t$. For systems with mixing, this probability tends to the proportion of $\mathcal{D}$ as $t \rightarrow \infty$, namely, to $\mu(\mathcal{D}) / \mu\left(\mathbb{T}^{n}\right)$. For systems with diffusion, the probability simply tends to a certain limit, and therefore one can speak of the limit state of statistical equilibrium.

In system (1.7), each point has an individual recurrence property (this is the Kronecker theorem). The presence of diffusion implies that the recurrence property is nonuniform. For $n \geq 3$, (3.3) does not hold. In relation to this remark, we mention the following important result from [24]: There exist incommensurable numbers $\alpha_{1}, \ldots, \alpha_{n}$, where $n \geq 3$, and an analytic function $R: \mathbb{T}^{n} \rightarrow \mathbb{R}$ such that (1.7) is a system with mixing.

If the numbers $\alpha_{2}, \ldots, \alpha_{n}$ have rational expressions in terms of $\alpha_{1}$, then all trajectories of system (1.7) are closed and the system cannot therefore be ergodic. In particular, mixing is out of the question. However, since the rotation periods on different trajectories do not coincide in the general case, there is no uniform recurrence and so diffusion may occur.

Thus, suppose that

$$
\alpha_{2}=\frac{p_{2}}{q_{2}} \alpha_{1}, \ldots, \alpha_{n}=\frac{p_{n}}{q_{n}} \alpha_{1}
$$

where $p_{j}$ and $q_{j}, j \geq 2$, are coprime integers. All trajectories are periodic; they can be indexed by the points of the $(n-1)$-torus $\mathbb{T}^{n-1}=\left\{u_{1}=0\right\} \subset \mathbb{T}^{n}$, which intersects all the trajectories. Each closed trajectory has countably many periods, but all of them are multiples of one period. In general, the periods $P$ of closed trajectories can be chosen so that they smoothly depend on the trajectories. Thus, we have a positive period function $P: \mathbb{T}^{n-1} \rightarrow \mathbb{R}$ for closed trajectories. If the function $R$ is infinitely differentiable (analytic), then so is $P$. Of course, the function $P$ has critical points (e.g., points of maximum and minimum). The further results substantially depend on whether these points are nondegenerate or not.

Theorem 9. If the integers $q_{2}, \ldots, q_{n}$ are pairwise coprime and $P: \mathbb{T}^{n-1} \rightarrow \mathbb{R}$ is a Morse function (all of its critical points are nondegenerate), then system (1.7) has the diffusion property (i.e., for any $F$ and $G$ in $L_{2},(3.1)$ has a limit as $\left.t \rightarrow \infty\right)$. 
Remark 1. It is unclear to what extent the assumption that $\left(q_{i}, q_{j}\right)=1$ for $i<j$ is essential. For $n=2$, it holds trivially. Apparently, the assumption that the critical points of $P$ are nondegenerate can be replaced by the requirement that they have finite multiplicity.

Now, take functions $F$ and $G$ in $L_{2}\left(\mathbb{T}^{n}\right)$ and consider the sequences of rational numbers

$$
\left(\frac{p_{2}}{q_{2}}\right)_{s}, \ldots,\left(\frac{p_{n}}{q_{n}}\right)_{s}, \quad s \geq 1
$$

determined by (3.4), which converge to $\nu_{2}, \ldots, \nu_{n}$, respectively, as $s \rightarrow \infty$. Obviously, for any $\nu_{2}, \ldots, \nu_{n}$, the convergent sequences (3.5) can be selected so that the denominators are pairwise coprime. Then the function $K(t)$ defined by (3.1) depends on $s$ (to be more precise, the phase flow of system (1.7) with the coefficients $\alpha_{j}$ defined by (3.4) and (3.5) depends on $s$ ); we denote this function by $K_{s}(t)$. Each set of rational numbers (3.5) corresponds to its own period function $P_{s}$ of closed trajectories. If the critical points of $P_{s}$ are nondegenerate, then (by Theorem 9) $K_{s}(t) \rightarrow \varkappa_{s}$, where $\varkappa_{s}=$ const, as $t \rightarrow \infty$.

Theorem 10. If the numbers $1, \nu_{2}, \ldots, \nu_{n}$ are incommensurable and $P_{s}$ is a Morse function for all $s \geq 1$, then

$$
\lim _{s \rightarrow \infty} \varkappa_{s}=\int_{\mathbb{T}^{n}} F R d^{n} u \int_{\mathbb{T}^{n}} G R d^{n} u / \int_{\mathbb{T}^{n}} R d^{n} u .
$$

This property can be called limit mixing. It is observed in computer simulations: when incommensurable numbers $\alpha_{j} / \alpha_{1}, j \geq 2$, are replaced by irreducible fractions $p_{j} / q_{j}$ with large $p_{j}$ and $q_{j}$, system (1.7) becomes virtually indistinguishable from a system with mixing.

Theorems 9 and 10 were stated in [25] for $n=2$. To conclude this section, we make a few remarks.

A. The period function $P$ can be obtained by averaging the function $R$ over the closed trajectories of system (1.7). Indeed, we have

$$
R(u) d u_{j}=\alpha_{j} d t, \quad j \geq 1 .
$$

Since $d u_{j} / \alpha_{j}=d u_{1} / \alpha_{1}$, these relations are equivalent to the single equation

$$
R(u) d u_{1}=\alpha_{1} d t
$$

Taking into account (3.4), we see that the closed trajectories of system (1.7) have the form

$$
u_{j}=\frac{p_{j}}{q_{j}} u_{1}+c_{j}, \quad c_{j}=\text { const }, \quad j \geq 2 .
$$

According to (3.7), we have

$$
\int_{0}^{k} R\left(u, \frac{p_{2}}{q_{2}} u+c_{2}, \ldots, \frac{p_{n}}{q_{n}} u+c_{n}\right) d u=\alpha_{1} P
$$

where $P$ is the period of a closed trajectory and $k$ is an integer equal to the number of rotations of closed trajectories along the cycle $\Gamma_{1}$. Clearly, the numbers $c_{2}, \ldots, c_{n} \bmod 1$ (the points of $\mathbb{T}^{n-1}$ ) index closed trajectories, and the period is a function of $c_{2}, \ldots, c_{n}$.

B. Is there a relationship between the limit mixing property (see Theorem 10) and the conditions for system (1.7) to be irreducible to system (2.2) (Theorems 3 and 4)? To better understand 
this question, consider the case in which $R$ is a trigonometric polynomial (i.e., the Fourier series of $R$ contains only finitely many harmonics). In this case, equations (1.7) reduce to (2.2) for any incommensurable set of $\alpha_{1}, \ldots, \alpha_{n}$. Now, let us approximate the ratios $\alpha_{2} / \alpha_{1}, \ldots, \alpha_{n} / \alpha_{1}$ by a sequence of rationals (3.5). It is easy to show that if $\alpha_{1}, \ldots, \alpha_{n}$ are incommensurable, then, starting with some $s$, the period function (3.8) is identically constant. Therefore, if $R$ is a trigonometric polynomial, then the conditions of Theorem 10 are surely violated. Roughly speaking, Theorem 10 is valid only for those functions $R$ whose Fourier series contain "almost all" harmonics. Precisely for such $R$, equations (1.7) do not reduce to (2.2) for an appropriate nonresonant set of $\alpha_{1}, \ldots, \alpha_{n}$. However, this statement needs to be rendered more rigorous.

In any case, Theorem 10 does not contradict the absence of mixing in system (1.7) for strongly incommensurable sets of $\alpha_{1}, \ldots, \alpha_{n}$. If (1.7) reduces to (2.2), then the function (3.1) oscillates and, therefore, has no limit as $t \rightarrow \infty$ in the standard sense. However, it converges to (3.2) in the sense of Cesaro. Theorem 10 shows that under some additional conditions, the Cesaro convergence can be replaced by the convergence for a sequence of systems of the form (1.7) with rational $\alpha_{j} / \alpha_{1}$.

C. A similar object is encountered in the Poincaré theory of birth of nondegenerate periodic solutions under perturbations of nondegenerate completely integrable systems with compact level surfaces of the first integrals [26]. The key idea is to average the perturbing function over the closed trajectories of a completely resonant invariant torus of the unperturbed system. If the result of averaging is a Morse function on the family of closed trajectories, then, under perturbation, this family gives rise to a pair of nondegenerate periodic trajectories.

D. Theorems 9 and 10 can be somewhat generalized. To this end, consider a sequence of special resonant tori that tend to a torus with incommensurable $\alpha_{1}, \ldots, \alpha_{n}$. The resonant tori are determined by $l$ relations of the form

$$
k_{11} \alpha_{1}+\ldots+k_{1 n} \alpha_{n}=\ldots=k_{l 1} \alpha_{1}+\ldots+k_{l n} \alpha_{n}=0
$$

with linearly independent integer vectors

$$
\left(k_{11}, \ldots, k_{1 n}\right), \ldots,\left(k_{l 1}, \ldots, k_{l n}\right) .
$$

Using (3.9), we can linearly express $n-l$ frequencies $\alpha_{j}$ in terms of the remaining frequencies; the remaining $l$ frequencies are assumed to be strongly incommensurable. Under these conditions, the torus $\mathbb{T}^{n}$ is fibered into an $(n-l)$-parameter family of invariant $l$-tori, and equations (1.7) can be reduced to the form (2.2) on each of these tori.

Next, we average the function $R$ over the strongly nonresonant $l$-tori. The most important condition for what follows is that, after averaging, we obtain a Morse function on the $(n-l)$-torus.

Under the above assumptions, equalities (3.9) imply that system (1.7) has the diffusion property (this is a generalization of Theorem 9). Moreover, if each of the resonant tori in the sequence specified above satisfies these conditions, then the limit equality (3.6) from Theorem 10 is valid.

\section{PROOFS}

$1^{\circ}$. To prove Theorem 2 , consider the transformation $u_{1}, \ldots, u_{n} \mapsto w_{1}, \ldots, w_{n}$ mod 1 defined by

$$
w_{j}=u_{j}+\frac{\alpha_{j}}{\langle R\rangle} f\left(u_{1}, \ldots, u_{n}\right), \quad 1 \leq j \leq n,
$$

where $\langle R\rangle$ is the mean value of the periodic function $R(u)=1 / r(u)$ over the torus $\mathbb{T}^{n}=\{u \bmod 1\}$ and $f: \mathbb{T}^{n} \rightarrow \mathbb{R}$ satisfies the linear partial differential equation

$$
\sum \frac{\partial f}{\partial u_{j}} \alpha_{j}=R-\langle R\rangle
$$


This equation is easily solved by the Fourier method:

$$
f(u)=\sum_{m}^{\prime} \frac{R_{m}}{2 \pi i(m, \alpha)} e^{2 \pi i(m, u)}, \quad m \in \mathbb{Z}^{n} \backslash\{0\},
$$

where $R_{m}$ are the Fourier coefficients of the function $R$. Since the numbers $|(m, \alpha)|$ obey the power estimate (2.1) and the Fourier coefficients of an infinitely differentiable (analytic) function decrease faster than any degree of $|m|$ (at an exponential rate), it follows that this series converges to a smooth (analytic) function.

In the new variables $w \bmod 1$, equations (1.7) take the form

$$
\dot{w}_{j}=\dot{u}_{j}+\frac{\alpha_{j}}{\langle R\rangle} \sum \frac{\partial f}{\partial u_{k}} \frac{\alpha_{k}}{R}=\frac{\alpha_{j}}{R}+\frac{\alpha_{j}}{\langle R\rangle} \frac{R-\langle R\rangle}{R}=\frac{\alpha_{j}}{\langle R\rangle}=\omega_{j} .
$$

The last equality follows from (1.20).

It remains to be shown that the change of variables (4.1) defines a diffeomorphism of the torus. It is easy to calculate the Jacobian of the transformation (4.1):

$$
\frac{\partial\left(w_{1}, \ldots, w_{n}\right)}{\partial\left(u_{1}, \ldots, u_{n}\right)}=\frac{R}{\langle R\rangle} .
$$

Therefore, the change (4.1) is nondegenerate. Let us prove that it is one-to-one. First, the transformation (4.1) leaves all lines on $\mathbb{T}^{n}$ of the form

$$
t \mapsto u^{0}+\alpha t, \quad t \in \mathbb{R},
$$

invariant. Second, the components of the vector function

$$
w(t)=u^{0}+\alpha t+\frac{\alpha}{\langle R\rangle} f\left(u^{0}+\alpha t\right)
$$

monotonically depend on $t$ :

$$
\dot{w}=\frac{\alpha}{\langle R\rangle} R\left(u^{0}+\alpha t\right) .
$$

This completes the proof of Theorem 2 .

$2^{\circ}$. Let us prove Theorem 3. For simplicity, we assume that $\alpha_{1}=1$. Consider a solution of (1.7) with zero initial condition for $t=0$. Obviously, $u_{j}=\alpha_{j} u_{1}$. Since $\dot{u}_{1}=R^{-1}(u)$, we have

$$
\frac{d t}{d u_{1}}=R\left(\alpha_{1} u_{1}, \alpha_{2} u_{2}, \ldots, \alpha_{n} u_{n}\right)
$$

on this solution. Let $R=\langle R\rangle+\widetilde{R}$, where $\langle\widetilde{R}\rangle=0$. Then,

$$
t=\langle R\rangle u_{1}+\int_{0}^{u_{1}} \widetilde{R}\left(\alpha_{1} \tau, \ldots, \alpha_{n} \tau\right) d \tau .
$$

Since the numbers $\alpha_{1}, \ldots, \alpha_{n}$ are incommensurable and $\langle\widetilde{R}\rangle=0$, it follows from the Weyl theorem that the integral on the right-hand side of $(4.2)$ is $o\left(u_{1}\right)$.

Now, suppose that there exists a continuous invertible transformation $\psi: u \mapsto w$ of the torus (with a continuous inverse $\psi^{-1}$ ) that conjugates the phase flows of the systems of differential equations (1.7) and (2.2). Then,

$$
g_{v}^{t}=\psi^{-1} g_{\omega}^{t} \psi
$$


and

$$
u(t)=g_{v}^{t}(u)=\psi^{-1} g_{\omega}^{t} \psi(u)=\psi^{-1} g_{\omega}^{t}(w)=\psi^{-1}(w(t)) .
$$

Since $\psi$ is a self-homeomorphism of $\mathbb{T}^{n}, \exp \left\{2 \pi i u_{k}(t)\right\}$ are single-valued continuous functions of $w_{1}=w_{1}^{0}+\omega_{1} t, \ldots, w_{n}=w_{n}^{0}+\omega_{n} t$. Applying the theorem about the argument of a conditionally periodic function, we obtain, in particular,

$$
u_{1}=\lambda t+f(t), \quad f(t)=O(1) .
$$

Since $\dot{u}_{1} \neq 0$, there exists an inverse function $t=t\left(u_{1}\right)$, and

$$
t=\frac{u_{1}}{\lambda}+O(1)
$$

Indeed, let $t=u_{1} / \lambda+g\left(u_{1}\right)$. Then,

$$
t=\frac{\lambda t+f(t)}{\lambda}+g(\lambda t+f(t))
$$

This implies

$$
g\left(u_{1}\right)=-\frac{f\left(u_{1} / \lambda+g\left(u_{1}\right)\right)}{\lambda}=O(1) .
$$

Comparing (4.2) and (4.3), we obtain $\lambda=\langle R\rangle^{-1}$, and the integral in (4.2) is bounded, as required.

$3^{\circ}$. If the integral in (4.2) is bounded, then it is a conditionally periodic function of $u_{1}$. Therefore, its Fourier coefficient corresponding to the harmonic

$$
\exp \left\{2 \pi i\left(m_{1} \alpha_{1}+\ldots+m_{n} \alpha_{n}\right) u_{1}\right\}, \quad \sum m_{j}^{2} \neq 0
$$

is equal to

$$
\frac{R_{m}}{2 \pi i(m, \alpha)}
$$

The inequality

$$
\sum^{\prime}\left|\frac{R_{m}}{(m, \alpha)}\right|^{2}<\infty
$$

which is a necessary condition for reducibility, is the Bessel inequality for the Fourier coefficients of a conditionally periodic function. To complete the proof of Theorem 4, it suffices to recall that the sets of numbers $\alpha_{1}, \ldots, \alpha_{n}$ and $\omega_{1}, \ldots, \omega_{n}$ are proportional.

$4^{\circ}$. Theorem 5 easily follows from Weyl's uniform distribution theorem. By virtue of (1.20), it is sufficient to consider differential equations of the form (1.7). Let us perform a change of time $t \mapsto \tau$ along trajectories of (1.7) by the formula

$$
d \tau=\frac{d t}{R(u)}
$$

Then system (1.7) takes the form

$$
\frac{d u_{j}}{d \tau}=\alpha_{j}
$$

A general solution to this system is given by $u_{j}=u_{j}^{0}+\alpha_{j} \tau$. Therefore,

$$
t=\int_{0}^{\tau} R\left(u_{1}^{0}+\alpha_{1} s, \ldots, u_{n}^{0}+\alpha_{n} s\right) d s .
$$


Since the numbers $\alpha_{1}, \ldots, \alpha_{n}$ are incommensurable, it follows from Weyl's theorem that $t=$ $\langle R\rangle \tau+o(\tau)$. Therefore,

$$
\tau=\frac{t}{\langle R\rangle}+o(t)
$$

Next,

$$
\frac{1}{T} \int_{0}^{T} f\left(u\left(t, u^{0}\right)\right) d t=\frac{1}{T} \int_{0}^{T^{\prime}} f\left(\alpha \tau+u^{0}\right) R\left(\alpha \tau+u^{0}\right) d \tau, \quad T=\int_{0}^{T^{\prime}} R\left(\alpha \tau+u^{0}\right) d \tau
$$

This equality does not change when both integrals on its right-hand side are divided by $T^{\prime}$. Clearly, $T^{\prime} \rightarrow \infty$ as $T \rightarrow \infty$. Applying again Weyl's theorem, we obtain

$$
\lim _{T \rightarrow \infty} \frac{1}{T} \int_{0}^{T} f\left(u\left(t, u^{0}\right)\right) d t=\int_{\mathbb{T}^{n}} f R d^{n} u / \int_{\mathbb{T}^{n}} R d^{n} u
$$

as required.

$5^{\circ}$. Let us prove Theorem 6. First, suppose that $\langle f\rangle=0$. By (4.5), we have

$$
\int_{0}^{T} f\left(u\left(t, u^{0}\right)\right) d t=\int_{0}^{T^{\prime}} \varphi\left(\alpha \tau+u^{0}\right) d \tau
$$

where $\varphi=f R$. Since $\langle f\rangle=0$, it follows that

$$
\int_{\mathbb{T}^{n}} \varphi d^{n} u=\int_{\mathbb{T}^{n}} f R d^{n} u=0
$$

According to the generalized Bohl theorem proved in [19], there exists a $u^{0} \in \mathbb{T}^{n}$ such that

(a) $\varphi\left(u^{0}\right)=0$;

(b) the integral (4.6) is a nonnegative (nonpositive) function of $T^{\prime}$.

Since $R \neq 0$, property (a) is equivalent to the condition $f\left(u^{0}\right)=0$. On the other hand, since $T$ is a monotone function of $T^{\prime}$, the integral on the left-hand side of (4.6) is also nonnegative (nonpositive) for all values of $T$.

If $\langle f\rangle \neq 0$, then $f$ should be replaced by $f-\langle f\rangle$.

$6^{\circ}$. Theorem 7 follows from the well-known results on the integrals of conditionally periodic functions (see [19] for $n=2$ and [27] for $n>2$ ) and from the arguments used in the proofs of Theorems 5 and 6 .

$7^{\circ}$. Theorem 8 follows from a general result on ergodic transformations [28]. This result was stated and proved in [28] for discrete transformations, but the proof for continuous flows is based on the same ideas.

$8^{\circ}$. Let us prove Theorem 9. Suppose that the numbers $\alpha_{1}, \ldots, \alpha_{n}$ satisfy (3.4). We pass to new variables $z_{1}, \ldots, z_{n}$ by making the change

$$
z_{1}=p_{2} u_{1}-q_{2} u_{2}, \quad \ldots, \quad z_{n-1}=p_{n} u_{1}-q_{n} u_{n}, \quad z_{n}=s_{1} u_{1}+s_{2} u_{2}+\ldots+s_{n} u_{n}
$$


where $s_{j}$ are integers and the $n \times n$ matrix

$$
\left(\begin{array}{ccccc}
p_{2} & -q_{2} & 0 & \ldots & 0 \\
\ldots \ldots & \ldots & \ldots \ldots & \ldots & \ldots \\
p_{n} & 0 & 0 & \ldots & -q_{n} \\
s_{1} & s_{2} & s_{3} & \ldots & s_{n}
\end{array}\right)
$$

is unimodular.

Let us show that if $\left(p_{i}, q_{i}\right)=1$ and $\left(q_{i}, q_{j}\right)=1$ for all $i<j$, then we can indeed render the matrix (4.7) unimodular by suitably selecting the integers $s_{j}$. The determinant of this matrix equals

$$
s_{1} k_{1}+s_{2} k_{2}+\ldots+s_{n} k_{n}
$$

where

$$
k_{1}=q_{2} q_{3} \ldots q_{n}, \quad k_{2}=p_{2} q_{3} \ldots q_{n}, \quad \ldots, \quad k_{n}=p_{n} q_{2} \ldots q_{n-1} .
$$

It is sufficient to show that the integers $k_{1}, \ldots, k_{n}$ are coprime. Suppose that a prime $p$ divides all $k_{j}: p \mid k_{j}$. Since $p \mid k_{1}$, one of the numbers $q_{2}, \ldots, q_{n}$, say $q_{2}$, is divisible by $p$. Since $p \mid k_{2}$ and $q_{3}, \ldots, q_{n}$ are coprime to $q_{2}$, it follows that $p \mid p_{2}$. But this contradicts the assumption that the fraction $p_{2} / q_{2}$ is irreducible.

In the new variables, system (1.7) takes the form

$$
\dot{z}_{1}=\ldots=\dot{z}_{n-1}=0, \quad \dot{z}_{n}=\Omega / R(z),
$$

where $\Omega=\sum s_{j} \alpha_{j} \neq 0$ and $R(z)$ is the density of the invariant measure in the angular variables $z_{1}, \ldots, z_{n}$.

We can further simplify system (4.8) by eliminating the dependence of the right-hand side on the coordinate $z_{n}$. For this purpose, we introduce new angular variables $y_{1}, \ldots, y_{n-1}, x \bmod 1$ defined by

$$
y_{1}=z_{1}, \quad \ldots, \quad y_{n-1}=z_{n-1}, \quad x=\frac{1}{\lambda} \int_{0}^{z_{n}} R\left(s, y_{1}, \ldots, y_{n-1}\right) d s
$$

where

$$
\lambda=\int_{0}^{1} R\left(s, y_{1}, \ldots, y_{n-1}\right) d s \neq 0 .
$$

This change is nondegenerate because

$$
\frac{\partial\left(y_{1}, \ldots, y_{n-1}, x\right)}{\partial\left(z_{1}, \ldots, z_{n-1}, z_{n}\right)}=\frac{R}{\lambda}
$$

In the new variables, equations (4.8) take the form

$$
\dot{y}=0, \quad \dot{x}=\omega(y),
$$

where $y=\left(y_{1}, \ldots, y_{n-1}\right)$ is a point of the $(n-1)$-torus and

$$
\omega(y)=\Omega / \lambda(y) .
$$

According to (4.9), the invariant measure $R(z) d^{n} z$ is expressed as $\lambda(y) d^{n-1} y d x$ in the variables $x, y \bmod 1$, the points $y \in \mathbb{T}^{n-1}$ index the closed orbits, and the period function $P$ of the closed 
orbits equals $\lambda(y) / \Omega$. Clearly, the smooth functions $y \mapsto P(y)$ and $y \mapsto \omega(y)$ are Morse functions simultaneously. A general solution to system (4.10) has the form

$$
x=\omega(y) t+x_{0}, \quad y=y_{0}, \quad x_{0}, y_{0}=\text { const. }
$$

Let $A$ and $B$ be functions on $\mathbb{T}^{n}=\{x, y \bmod 1\}$ that are square integrable with respect to the invariant measure $\lambda d^{n-1} y d x$ (for definiteness, we assume that $\lambda>0$ ). Set

$$
a(y)=\int_{0}^{1} A(x, y) d x \quad \text { and } \quad b(y)=\int_{0}^{1} B(x, y) d x .
$$

Clearly, the functions $a$ and $b$ belong to $L_{2}\left(\mathbb{T}^{n-1}\right)$. By Fubini's theorem, the functions $A$ and $B$ (as functions of $x$ ) are square integrable for almost all $y \in \mathbb{T}^{n-1}$. Let

$$
\sum a_{m}(y) e^{2 \pi i m x} \quad \text { and } \quad \sum b_{m}(y) e^{2 \pi i m x}
$$

be their Fourier series. Obviously, $a_{m}, b_{m} \in L_{2}\left(\mathbb{T}^{n-1}\right)$ for all $m \in \mathbb{Z}$.

We will prove an assertion somewhat stronger than that of Theorem 9, namely,

$$
\lim _{t \rightarrow \infty} \int_{\mathbb{T}^{n}} A(x-\omega(y) t, y) B(x, y) \lambda(y) d x d^{n-1} y=\int_{\mathbb{T}^{n-1}} a b \lambda d^{n-1} y .
$$

The integral on the left-hand side of (4.11) equals

$$
\int_{\mathbb{T}^{n-1}} \sum a_{-m} b_{m} \lambda e^{-2 \pi i m \omega(y) t} d^{n-1} y .
$$

First, let us show that this series converges (uniformly in $t$ ). For this purpose, it suffices to prove that

$$
\sum_{m} \int_{\mathbb{T}^{n-1}}\left|a_{-m} b_{m}\right| \lambda d^{n-1} y<\infty
$$

Indeed,

$$
\left|a_{-m} b_{m}\right|+\left|a_{m} b_{-m}\right| \leq a_{-m} a_{m}+b_{-m} b_{m}
$$

On the other hand, the Bessel inequality for functions in $L_{2}$ implies

$$
\sum a_{-m} a_{m} \leq \int_{0}^{1} A^{2} d x \quad \text { and } \quad \sum b_{-m} b_{m} \leq \int_{0}^{1} B^{2} d x .
$$

Therefore, the left-hand side of (4.13) cannot exceed

$$
\int_{\mathbb{T}^{n}}\left(A^{2}+B^{2}\right) \lambda d x d^{n-1} y
$$

By the Lebesgue theorem (in view of (4.13)), the integrand in (4.12) is indeed integrable, and the integral (4.12) can be represented as

$$
\int_{\mathbb{T}^{n-1}} a b \lambda d^{n-1} y+\sum_{m \neq 0} \int_{\mathbb{T}^{n-1}} a_{-m} b_{m} \lambda e^{-2 \pi i m \omega(y) t} d^{n-1} y .
$$


Each term in the second sum tends to zero as $t \rightarrow \infty$. Indeed, the product $a_{-m} b_{m} \lambda$ is an integrable function on $\mathbb{T}^{n-1}$, and the function $y \mapsto \omega(y)$ has only nondegenerate critical points on $\mathbb{T}^{n-1}$. Therefore, the required assertion follows from a well-known general result of the theory of Fourier transforms (see, e.g., [29]).

Since the series (4.13) converges, the infinite sum of the terms with numbers $|m| \geq N(\varepsilon)$ in (4.14) can be made smaller than $\varepsilon / 2$. The remaining terms with $m \neq 0$ and $|m|<N(\varepsilon)$ tend to zero as $t \rightarrow \infty$, and their sum is therefore less than $\varepsilon / 2$ for sufficiently large $t$, as required.

$9^{\circ}$. It remains to prove Theorem 10. Using formula (4.9) for the Jacobian of the transformation $z_{1}, \ldots, z_{n} \mapsto y_{1}, \ldots, y_{n-1}, x \bmod 1$ and equations (4.10), we obtain

$$
K_{s}(t)=\int_{\mathbb{T}^{n}} F\left(x-\omega_{s}(y) t, y\right) G(x, y) \lambda_{s}(y) d x d^{n-1} y
$$

where $\omega_{s}(y)=\Omega_{s} / \lambda_{s}(y)$ with $\Omega_{s}=$ const $\neq 0$. Since $\lambda_{s}$ is a Morse function, it follows from Theorem 9 that

$$
K_{s}(t) \rightarrow \varkappa_{s}=\int_{\mathbb{T}^{n-1}} f_{s}(y) g_{s}(y) \lambda_{s}(y) d^{n-1} y
$$

as $t \rightarrow \infty$. Here, $f_{s}$ and $g_{s}$ are the mean values of $F_{s}$ and $G_{s}$ along the closed trajectories of system (1.7) in which the coefficients $\alpha_{1}, \ldots, \alpha_{n}$ satisfy (3.4) with rational coefficients (3.5). We also use the fact that $\lambda_{s}$ do not depend on $x$. Moreover, $\lambda_{s}$ are uniformly bounded, and

$$
\int_{\mathbb{T}^{n-1}} \lambda_{s}(y) d^{n-1} y=\int_{\mathbb{T}^{n}} R(u) d^{n} u
$$

Let us show that

$$
\varkappa_{s} \rightarrow\langle F\rangle\langle G\rangle \int_{\mathbb{T}^{n}} R d^{n} u
$$

as $s \rightarrow \infty$. It is well known that continuous functions are everywhere dense in $L_{2}$. Therefore, the functions $F$ and $G$ can be approximated with any accuracy by continuous functions $\widetilde{F}$ and $\widetilde{G}$.

As $s \rightarrow \infty$, the numbers $\alpha_{2} / \alpha_{1}=\left(p_{2} / q_{2}\right)_{s}, \ldots, \alpha_{n} / \alpha_{1}=\left(p_{n} / q_{n}\right)_{s}$ tend to $\nu_{2}, \ldots, \nu_{n}$, and the numbers $1, \nu_{2}, \ldots, \nu_{n}$ are incommensurable; hence, according to [11, Chapter VII], $\widetilde{f}_{s}$ and $\widetilde{g}_{s}$ tend to the spatial averages of the continuous functions $\widetilde{F}$ and $\widetilde{G}$, respectively; i.e.,

$$
\widetilde{f}_{s}(y) \rightarrow\langle\widetilde{F}\rangle=\int_{\mathbb{T}^{n}} \widetilde{F} R d^{n} u / \int_{\mathbb{T}^{n}} R d^{n} u \quad \text { and } \quad \widetilde{g}_{s}(y) \rightarrow\langle\widetilde{G}\rangle=\int_{\mathbb{T}^{n}} \widetilde{G} R d^{n} u / \int_{\mathbb{T}^{n}} R d^{n} u
$$

uniformly in $y \in \mathbb{T}^{n-1}$.

Let us estimate the difference of the integrals

$$
\int_{\mathbb{T}^{n-1}} f_{s} g_{s} \lambda_{s} d^{n-1} y \quad \text { and } \quad \int_{\mathbb{T}^{n-1}} \widetilde{f}_{s} \widetilde{g}_{s} \lambda_{s} d^{n-1} y
$$

using the identity $f_{s} g_{s}=f_{s} g_{s}-\widetilde{f}_{s} g_{s}+\widetilde{f}_{s} g_{s}-\widetilde{f}_{s} \widetilde{g}_{s}+\widetilde{f}_{s} \widetilde{g}_{s}$. By the Cauchy-Schwarz inequality,

$$
\left[\int_{\mathbb{T}^{n-1}}\left(f_{s}-\tilde{f}_{s}\right) g_{s} \lambda_{s} d^{n-1} y\right]^{2} \leq \int_{\mathbb{T}^{n-1}}\left(f_{s}-\widetilde{f}_{s}\right)^{2} \lambda_{s} d^{n-1} y \int_{\mathbb{T}^{n-1}} g_{s}^{2} \lambda_{s} d^{n-1} y .
$$


Since $f_{s}, \widetilde{f}_{s}$, and $g_{s}$ are obtained by averaging the functions $F, \widetilde{F}$, and $G$ along closed trajectories, it follows from the Bessel inequality that the integrals on the right-hand side of (4.20) do not exceed

$$
\int_{\mathbb{T}^{n}}(F-\widetilde{F})^{2} R d^{n} u \quad \text { and } \quad \int_{\mathbb{T}^{n}} G^{2} R d^{n} u,
$$

respectively. Therefore, the integrals of the functions $f_{s} g_{s}$ and $\widetilde{f}_{s} g_{s}$ with respect to the measure $\lambda_{s} d^{n-1} y$ differ little from each other (uniformly in $s$ ) provided that $F$ and $\widetilde{F}$ are close in the metric of $L_{2}$. The integrals of the functions $\widetilde{f}_{s} g_{s}$ and $\widetilde{f}_{s} \widetilde{g}_{s}$ with respect to the same measure have the same property. Thus, if $\widetilde{F}$ and $\widetilde{G}$ tend to $F$ and $G$ (in the metric of $L_{2}$ ), then the difference of the integrals (4.19) tends to zero uniformly in $s$.

According to (4.16) and (4.18), we have

$$
\lim _{s \rightarrow \infty} \int_{\mathbb{T}^{n-1}} \widetilde{f}_{s} \widetilde{g}_{s} \lambda_{s} d^{n-1} y=\langle\widetilde{F}\rangle\langle\widetilde{G}\rangle \int_{\mathbb{T}^{n}} R d^{n} u .
$$

By the approximation assumption, $\langle\widetilde{F}\rangle$ and $\langle\widetilde{G}\rangle$ can be made arbitrarily close to $\langle F\rangle$ and $\langle G\rangle$. Therefore, the required relation (4.17) follows from (4.21). This completes the proof of the theorem.

\section{ACKNOWLEDGMENTS}

The author thanks A.V. Kochergin for useful discussions.

This work was supported by the Russian Foundation for Basic Research (project no. 05-01-01058) and by a grant of the President of the Russian Federation for the support of leading scientific schools (project no. NSh-1312.2006.1).

\section{REFERENCES}

1. H. Poincaré, Calcul des probabilités (Gauthier-Villars, Paris, 1912; Regulyarnaya i Khaoticheskaya Dinamika, Izhevsk, 1999).

2. A. N. Kolmogorov, "On Dynamical Systems with an Integral Invariant on the Torus," Dokl. Akad. Nauk SSSR 93 (5), 763-766 (1953).

3. A. N. Kolmogorov, "The General Theory of Dynamical Systems and Classical Mechanics," in Proceedings of the International Congress of Mathematicians, Amsterdam, the Netherlands, 1954 (Amsterdam, North-Holland, 1957), Vol. 1, pp. 315-333 [R.H. Abraham, Foundations of Mechanics (Benjamin, New York, 1967), Appendix D, pp. 263-279].

4. V. I. Arnold, "Polyintegrable Flows," Algebra Anal. 4 (6), 54-62 (1992) [St. Petersburg Math. J. 4, $1103-1110$ (1993)].

5. V. V. Kozlov, "Symmetries and Regular Behavior of Hamiltonian Systems," Chaos 6 (1), 1-5 (1996).

6. V. V. Kozlov and N. G. Moshchevitin, "Diffusion in Hamiltonian Systems," Chaos 8 (1), $245-247$ (1998).

7. Y. Nambu, "Generalized Hamiltonian Dynamics," Phys. Rev. D 7, 2405-2412 (1973).

8. S. P. Novikov, "The Hamiltonian Formalism and a Many-Valued Analogue of Morse Theory," Usp. Mat. Nauk 37 (5), 3-49 (1982) [Russ. Math. Surv. 37 (5), 1-56 (1982)].

9. I. A. Dynnikov and S. P. Novikov, "Topology of Quasi-periodic Functions on the Plane," Usp. Mat. Nauk 60 (1), 3-28 (2005) [Russ. Math. Surv. 60, 1-26 (2005)].

10. C. L. Siegel, "Note on Differential Equations on the Torus," Ann. Math. 46 (3), 423-428 (1945).

11. V. V. Kozlov, Methods of Qualitative Analysis in the Dynamics of a Rigid Body (Mosk. Gos. Univ., Moscow, 1980) [in Russian].

12. M. R. Herman, "Exemples de flots hamiltoniens dont aucune perturbation en topologie $C^{\infty}$ n'a d'orbites périodiques sur un ouvert de surfaces d'énergies," C. R. Acad. Sci. Paris, Sér. 1, 312 (13), 989-994 (1991).

13. D. V. Anosov, "On an Additive Functional Homology Equation Connected with an Ergodic Rotation of the Circle," Izv. Akad. Nauk SSSR, Ser. Mat. 37 (6), 1259-1274 (1973) [Math. USSR, Izv. 7, 1257-1271 (1973)]. 
14. A. Ya. Gordon, "Sufficient Condition for Unsolvability of the Additive Functional Homological Equation Connected with the Ergodic Rotation of a Circle," Funkts. Anal. Prilozh. 9 (4), 71-72 (1975) [Funct. Anal. Appl. 9, 334-336 (1975)].

15. A. M. Stepin, "On a Homological Equation of the Theory of Dynamical Systems," in Studies on the Theory of Functions of Many Real Variables (Yaroslav. Gos. Univ., Yaroslavl, 1982), pp. 106-117 [in Russian].

16. M. R. Herman, "Sur la conjugaison différentiable des difféomorphismes du cercle à des rotations," Publ. Math., Inst. Hautes Étud. Sci. 49, 5-233 (1979).

17. A. V. Rozhdestvenskii, "On the Additive Cohomological Equation and Time Change for a Linear Flow on the Torus with a Diophantine Frequency Vector," Mat. Sb. 195 (5), 115-156 (2004) [Sb. Math. 195, 723-764 (2004)].

18. P. Bohl, "Über eine Differentialgleichung der Störungstheorie," J. Reine Angew. Math. 131 (4), $268-321$ (1906).

19. V. V. Kozlov, "On Integrals of Quasiperiodic Functions," Vestn. Mosk. Univ., Ser. 1: Mat., Mekh., No. 1, $106-115$ (1978) [Moscow Univ. Math. Bull. 33 (1-2), 31-38 (1978)].

20. A. B. Katok, "Spectral Properties of Dynamical Systems with an Integral Invariant on the Torus," Funkts. Anal. Prilozh. 1 (4), 46-56 (1967) [Funct. Anal. Appl. 1, 296-305 (1967)].

21. A. V. Kochergin, "On the Absence of Mixing in Special Flows over the Rotation of a Circle and in Flows on a Two-Dimensional Torus," Dokl. Akad. Nauk SSSR 205 (3), 515-518 (1972) [Sov. Math. Dokl. 13, 949-952 (1972)].

22. V. I. Arnold, "Topological and Ergodic Properties of Closed 1-Forms with Incommensurable Periods," Funkts. Anal. Prilozh. 25 (2), 1-12 (1991) [Funct. Anal. Appl. 25, 81-90 (1991)].

23. Ya. G. Sinai and K. M. Khanin, "Mixing for Some Classes of Special Flows over Rotations of the Circle," Funkts. Anal. Prilozh. 26 (3), 1-21 (1992) [Funct. Anal. Appl. 26, 155-169 (1992)].

24. B. R. Fayad, "Analytic Mixing Reparametrizations of Irrational Flows," Ergodic Theory Dyn. Syst. 22 (2), 437-468 (2002).

25. V. V. Kozlov, "Diffusion in Systems with Integral Invariants on the Torus," Dokl. Akad. Nauk 381 (5), $596-598$ (2001) [Dokl. Math. 64 (3), 390-392 (2001)].

26. H. Poincaré, Les méthodes nouvelles de la Mécanique céleste (Gauthier-Villars, Paris, 1892), Vol. 1.

27. N. G. Moshchevitin, "Recurrence of the Integral of a Smooth Three-Frequency Conditionally Periodic Function," Mat. Zametki 58 (5), 723-735 (1995) [Math. Notes 58, 1187-1196 (1995)].

28. G. Halász, "Remarks on the Remainder in Birkhoff's Ergodic Theorem," Acta Math. Acad. Sci. Hung. 28 (3-4), 389-395 (1976).

29. Y. Colin de Verdière, "Nombre de points entiers dans une famille homothétique de domaines de $\mathbb{R}$," Ann. Sci. Éc. Norm. Super., Sér. 4, 10 (4), 559-575 (1977).

Translated by O. Sipacheva 